\title{
Competitive evaluation in male elite junior soccer players: entire match, replaced, and substitute players
}

\author{
Adriano Titton* \\ São Paulo Futebol Clube, President Laudo Natel Athletes Development Center, Cotia, Brazil
}

The purpose of this study was to analyze the mood states, recovery, displacement patterns, and rating of perceived exertion of elite junior soccer players during a national competition, considering substitutions in the matches. Before the games, the mood states and total quality recovery (TQR) were evaluated. During the games, the total distance (TD), low-speed distance (LSD), and high-speed distance were monitored. At the end of the matches, the rating of perceived exertion scale was used. The average and standard deviation were used to compare the match stages, soccer positions and influence of the substitutions. The significance level adopted was $5 \%$. In relation to the mood states, fatigue presented higher values $(P<0.05)$ for entire match and substitute players, and in the TQR, substitute players presented better recovery $(P<0.05)$ than entire match and replaced players. In the TD, shorter distances covered were observed $(P<0.05)$ in the first half, and the average of the midfielders was longer $(P<0.05)$ than that of the defenders. In the LSD, the midfielders covered longer distances $(P<0.05)$ than the strikers and defenders. It is possible to conclude that the substitutions have an impact on the player positions, match stages and maintenance of the intensity of the players.

Keywords: Soccer players, Mood states, Recovery, Global Positioning System, Rating of perceived exertion, Youth teams

\section{INTRODUCTION}

Soccer is a sport practiced in a context of high variability and randomness that occurs with high degree of unpredictability, and according to Turner and Stewart (2014), its motor stimulations have intermittent and acyclic characteristics.

Several studies have made progress in understanding the demands of soccer for training young soccer players (Aslan et al., 2012; Buchheit et al., 2010; Mendez-Villanueva et al., 2013; Saward et al., 2016), allowing individual and collective characterization of the players in relation to their displacements during the matches and showing that soccer players in their last years of training present displacement patterns that are proportionally similar to those of professional players (Saward et al., 2016).

It is also related in the literature that the physical performance of soccer players tends to decrease during the match due to many factors, such as accumulated fatigue, strategic trajectory, tactical choices, pace of the game, and other reasons (Hills et al., 2019).
Thus, an efficient strategy with the purpose of mitigating the effects of decreased performance of soccer players consists of substitutions made by coaches (Hills et al., 2018). However, only evaluating soccer displacements is not a comprehensive way to monitor the oscillations in performance during a competition.

Another method described in the literature (Fanchini et al., 2015; Foster et al., 2001) to assess the load imposed on the athlete in the face of the effort made in matches and training is the rating of perceived exertion (RPE). Nonetheless, during a competition, not only is monitoring the load imposed to the soccer players necessary for high performance but also monitoring the athlete's condition with regard to his recovery is fundamental during the competition period. Therefore, based on the concept of the use of psychophysical scales similar to the RPE applied before activities, Kenttä and Hassmém (1998) validated the total quality recovery (TQR), a reliable model with a simple application that makes monitoring athlete recovery possible.

Regarding the athlete's condition, the monitoring of psycho-
${ }^{*}$ Corresponding author: Adriano Titton (iD https://orcid.org/0000-0001-8330-3776 São Paulo Futebol Clube, President Laudo Natel Athletes Development Center, Avenida Doutor Odair Pacheco Pedroso, 1700, Cotia, 06717-642, Brazil E-mail: atitton1@hotmail.com

Received: March 17, 2020 / Accepted: April 1, 2020
This is an Open Access article distributed under the terms of the Creative Commons Attribution Non-Commercial License (https://creativecommons.org/licenses/by-nc/4.0/) which permits unrestricted non-commercial use, distribution, and reproduction in any medium, provided the original work is properly cited. 
logical variables such as mood state has helped to control the load, mediating the perception of fatigue. A psychological instrument that is commonly used and is effective and sensitive for detecting signs is the Brunel Model Scale (BRUMS) (Brandt et al., 2016). In view of this, the general purpose of the present study was to evaluate the different variables related to the competitive environment, to analyze the differences between the game positions and match stages and to evaluate the impact of substitutions during a competition in elite junior Brazilian soccer players.

\section{MATERIALS AND METHODS}

\section{Subjects}

The sample consisted of 17 male athletes who were outfield soccer players with an average age of $18.5 \pm 0.7$ years, height of $178.2 \pm 6.1 \mathrm{~cm}$, body mass of $72,7 \pm 6.3 \mathrm{~kg}$, and fat percentage of $6.9 \% \pm 1.5 \%$, which provided 89 observations of performance in competitive matches. All the soccer players had an average practice time of 4 years in soccer training, and they were members of youth teams in a big club in São Paulo - Brazil. Young outfield players who belonged to the club's junior team (under 20) and were registered in the competition of São Paulo Football Cup 2015 were included in the research. The formation used by the team was 1-4-3-3, with the goalkeeper, two defenders, two fullbacks, three midfielders, and three strikers. This study had the approval of the Ethics Committee of São Judas Tadeu University (Protocol 897.223) dated 12/03/2014, and all the volunteers and people responsible for them signed the informed consent form for participation in the study.

\section{Experimental procedures}

The data collection was performed in different stadiums where the matches occurred, and all of them were considered neutral by the organization. The soccer players participated in a competition during January over 20 days that included seven games (the first phase included three games, and the second phase included one game, followed by a round of 16 , a round of 8 , and a semifinal), as shown in Fig. 1. The result of the six first matches was victory, and there was a defeat in the last match, culminating in the elimi- nation of the team from the competition. All the substitutions made during the competition occurred in the second half of the match, and only one of the 19 substitutions carried out during seven games was carried out with defenders, while the other 18 were with midfielders and strikers.

\section{Instruments}

Before the games, the mood states of the athletes were monitored through BRUMS, and recovery was monitored with the TQR. During the match, the soccer players were individually monitored with a Global Positioning System (GPS) for the analysis of displacements, and at the end of the match, the RPE scale was applied (Fig. 2). All the athletes who participated in the study were already familiarized with the instruments used.

\section{Protocol on mood states}

The BRUMS test validated for Brazilian athletes by Brandt et al. (2016) was used for the assessment of mood states. This scale is used to assess six mood states: tension, depression, anger, vigor, fatigue, and confusion, allowing the verification of emotional variation associated with sport practices. Soccer players pointed out how they felt on the assessment day through a 5-point Likert-type scale: 0 (not at all), 1 (a little), 2 (moderately), 3 (quite a bit), and 4 (extremely). Subsequently, the answers were tabulated in a sheet that determined the six mood states and a total BRUMS score. However, in the present study, the only values analyzed were vigor and fatigue because they are the emotional states that are frequently modified in training and competitive games and were the object of study in a meta-analysis conducted by Slimani and Bragazzi (2017).

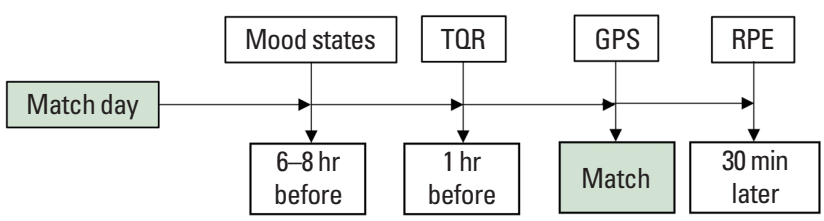

Fig. 2. Study experimental sequence. TQR, total quality recovery; GPS, Global Positioning System; RPE, rating of perceived exertion.

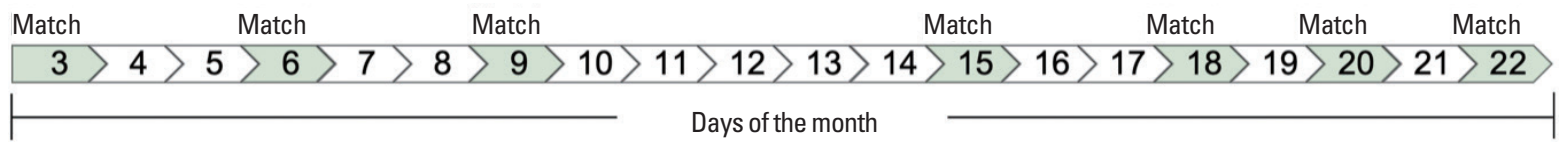

Fig. 1. Distribution of games throughout the days of the competitive period. 


\section{Recovery}

The TQR scale is graduated from 6 to 20 , with verbal anchors related to subjective perception of recovery. The closer the value is to 20 , the higher the perception of a good recovery, and the closer the value is to 6 , the less appropriate the perception of recovery (Kenttä and Hassmén, 1998). To apply the TQR method, the following question regarding their recovery was asked to the soccer players simultaneous with the presentation of the scale: "How are you?" The scale was applied $1 \mathrm{hr}$ before the games when the athletes were starting preparation for the matches in the dressing rooms.

\section{Physical performance}

A Qstarz BT-Q1300ST GPS Data Logger receiver $(1 \mathrm{~Hz})$ was put in an internal padded pocket on the shorts of each player in all games. The volume of displacements was evaluated through this device, and the total distance (TD) and two speed ranges, namely, low-speed distance (LSD) in displacements $\leq 15 \mathrm{~km} / \mathrm{hr}$ and highspeed distance (HSD) during sprints $>15 \mathrm{~km} / \mathrm{hr}$, were measured. To adjust the data, the absolute distance was measured in meters (m) divided by the played minutes (min), which is known as the relative distance $(\mathrm{m} / \mathrm{min})$, according to the suggestion of Cummins et al. (2013).

\section{Rating of perceived exertion}

Control of the internal load was achieved through the RPE of Borg (1982), with a scale graduated from 0 (no exertion) to 10 (maximal exertion). To apply the RPE method, the following question was asked to the players concomitantly with the presentation of the scale $30 \mathrm{~min}$ after the end of every game: "How did you perceive the effort made in the game?" All the RPE values were multiplied by the participation in minutes in the games, determin- ing a value (TRIMP) that represents the magnitude of the session in arbitrary units (AU) denominated the session-RPE (Foster et al., 2001).

\section{Statistical analysis}

A descriptive analysis of data was performed through averages and standard deviations comparing the results between match stages (first and second half) and game positions performed by the players. Analysis of variance (ANOVA) for mixed models (considering that the same athlete was evaluated several times) or ANOVA for a fixed factor were used for an inferential analysis always followed by multiple comparisons with Tukey method to detail where significant differences occurred. The analysis was performed with SAS 9 (Institute Inc., Cary, NC, USA). All results were evaluated using a significance level of $5 \%$.

\section{RESULTS}

Table 1 presents a descriptive analysis of the average of all the games of the competition, separated by the 1st half, 2nd half and total time using the average and standard deviation of the following variables: TD $(\mathrm{m} / \mathrm{min}), \operatorname{LSD}(\mathrm{m} / \mathrm{min}) \leq 15 \mathrm{~km} / \mathrm{hr}$, HSD $(\mathrm{m} /$ $\min )>15 \mathrm{~km} / \mathrm{hr}$, session-RPE, TQR, mood state vigor, and mood state fatigue.

Table 2 presents a descriptive analysis of the average of all the games of the competition separated according to the position performed (fullbacks, defenders, midfielders, or strikers) using the average and standard deviation of the following variables: TD $(\mathrm{m} / \mathrm{min})$, LSD $(\mathrm{m} / \mathrm{min}) \leq 15 \mathrm{~km} / \mathrm{hr}$, HSD $(\mathrm{m} / \mathrm{min})$, session-RPE, TQR, mood state vigor, and mood state fatigue.

Table 3 presents a descriptive analysis of the average of all games in the competition, separating entire match soccer players from

Table 1. Comparison of the first half, second half and total match average

\begin{tabular}{|c|c|c|c|c|c|c|}
\hline Variable & 1st half $(n=70)$ & 2nd half $(n=89)$ & $P$-value & $95 \% \mathrm{Cl}$ & Total average $(n=89)$ & $95 \% \mathrm{Cl}$ \\
\hline $\mathrm{TD}(\mathrm{m} / \mathrm{min})$ & $60 \pm 14^{*}$ & $63 \pm 29$ & 0.0035 & -4.6 to 9.3 & $110 \pm 11$ & $107.1-111.9$ \\
\hline $\operatorname{LSD}(\mathrm{m} / \mathrm{min}) \leq 15 \mathrm{~km} / \mathrm{hr}$ & $52 \pm 11^{*}$ & $54 \pm 24$ & 0.0037 & -3.6 to 7.5 & $94 \pm 8$ & $91.9-95.2$ \\
\hline $\mathrm{HSD}(\mathrm{m} / \mathrm{min})>15 \mathrm{~km} / \mathrm{hr}$ & $9 \pm 4^{*}$ & $9 \pm 6$ & 0.0121 & -1.2 to 2.0 & $16 \pm 5$ & 14.8-17.1 \\
\hline Session-RPE & & & & & $569 \pm 289$ & $508.3-630.1$ \\
\hline TQR & & & & & $15.3 \pm 1.0$ & $15.1-15.5$ \\
\hline Mood state vigor & & & & & $11.8 \pm 3.1$ & $11.2-12.5$ \\
\hline Mood state fatigue & & & & & $0.9 \pm 1.2$ & $0.6-1.1$ \\
\hline
\end{tabular}

Values are presented as the mean \pm standard deviation.

TD, total distance; LSD, low-speed distance; HSD, high-speed distance; RPE, rating of perceived exertion; TQR, total quality recovery.

Data from the 1st and 2nd halves and total match and time differences ( $P$-value) with 95\% confidence intervals (Cls).

*Significant difference from the 1 st to the 2 nd half of the match $(P>0.05)$. 
Table 2. Comparison of positions

\begin{tabular}{|c|c|c|c|c|c|}
\hline Variable & Fullbacks $(n=14)$ & Defenders $(n=15)$ & Midfielders $(n=28)$ & Strikers $(n=32)$ & $P$-value \\
\hline $\mathrm{TD}(\mathrm{m} / \mathrm{min})$ & $105 \pm 8$ & $102 \pm 5$ & $120 \pm 10$ & $106 \pm 11^{\text {a) }}$ & 0.0439 \\
\hline $\mathrm{LSD}(\mathrm{m} / \mathrm{min}) \leq 15 \mathrm{~km} / \mathrm{hr}$ & $91 \pm 6$ & $89 \pm 4^{b)}$ & $101 \pm 6$ & $90 \pm 7^{c)}$ & 0.0069 \\
\hline $\mathrm{HSD}(\mathrm{m} / \mathrm{min})>15 \mathrm{~km} / \mathrm{hr}$ & $14 \pm 3$ & $13 \pm 2$ & $19 \pm 6$ & $16 \pm 5$ & 0.4508 \\
\hline Session-RPE & $819 \pm 107$ & $703 \pm 211$ & $517 \pm 283$ & $443 \pm 295$ & 0.1461 \\
\hline TQR & $14.6 \pm 0.5$ & $14.7 \pm 1.0$ & $15.8 \pm 1.0$ & $15.4 \pm 1.1$ & 0.2209 \\
\hline Mood state vigor & $10.2 \pm 3.6$ & $14.2 \pm 2.5$ & $11.4 \pm 3.0$ & $11.8 \pm 2.8$ & 0.2994 \\
\hline Mood state fatigue & $1.7 \pm 1.3$ & $0.7 \pm 0.7$ & $0.7 \pm 1.3$ & $0.7 \pm 1.1$ & 0.3070 \\
\hline
\end{tabular}

Values are presented as the mean \pm standard deviation.

TD, total distance; LSD, low-speed distance; HSD, high-speed distance; RPE, rating of perceived exertion; TQR, total quality recovery.

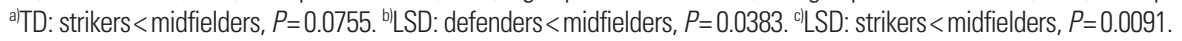

Table 3. Analysis of entire match, replaced, and substitute soccer players

\begin{tabular}{lcccr} 
Variable & $\begin{array}{c}\text { Entire match } \\
(\mathrm{n}=51)\end{array}$ & $\begin{array}{c}\text { Replaced } \\
(\mathrm{n}=19)\end{array}$ & $\begin{array}{c}\text { Substitute } \\
(\mathrm{n}=19)\end{array}$ & $P$-value \\
\hline $\mathrm{TD}(\mathrm{m} / \mathrm{min})$ & $106 \pm 10^{\mathrm{a}, \mathrm{b})}$ & $114 \pm 12$ & $115 \pm 12$ & 0.0016 \\
$\mathrm{LSD}(\mathrm{m} / \mathrm{min}) \leq 15 \mathrm{~km} / \mathrm{hr}$ & $92 \pm 7$ & $96 \pm 9$ & $96 \pm 8$ & 0.0353 \\
$\mathrm{HSD}(\mathrm{m} / \mathrm{min})>15 \mathrm{~km} / \mathrm{hr}$ & $14 \pm 4^{\mathrm{c}, \mathrm{d})}$ & $18 \pm 4$ & $19 \pm 7$ & 0.0004 \\
$\mathrm{Session}-\mathrm{RPE}$ & $769 \pm 116^{\mathrm{e}, \mathrm{f})}$ & $502 \pm 126^{\mathrm{g})}$ & $99 \pm 57$ & $<0.0001$ \\
$\mathrm{TQR}$ & $15.1 \pm 0.9^{\mathrm{h})}$ & $15.2 \pm 1.2^{\mathrm{i})}$ & $16.0 \pm 0.8$ & 0.0023 \\
Mood state vigor & $11.8 \pm 3.5$ & $12.2 \pm 3.1$ & $11.6 \pm 2.1$ & 0.8200 \\
Mood state fatigue & $1.1 \pm 1.4$ & $0.6 \pm 0.8$ & $\left.0.4 \pm 0.6^{\mathrm{j}}\right)$ & 0.0387 \\
\hline
\end{tabular}

Values are presented as the mean \pm standard deviation.

TD, total distance; LSD, low-speed distance; HSD, high-speed distance; RPE, rating of perceived exertion; TQR, total quality recovery.

alTD: entire match < replaced $P=0.0168$. ${ }^{\text {bTTD }}$ : entire match $<$ substitute $P=0.0068$. ${ }^{c}$ HSD: entire match $<$ replaced $P=0.0189$. ${ }^{\text {d) }} H S D$ : entire match $<$ substitute $P=0.0011$. elSession-RPE: entire match $>$ replaced $P \leq 0.0001$. ${ }^{\text {fl }}$ Session-RPE: entire match> substitute $P \leq 0.0001$. ${ }^{\text {I) }}$ Session-RPE: replaced $>$ substitute $P \leq 0.0001$. ${ }^{\text {h/TOR: entire }}$ match<substitute $P=0.0016$. ITQR: entire match<substitute $P=0.0384$. "Mood states fatigue: entire match $>$ substitute $P=0.0460$.

replaced and substitute players using the average and standard deviation of the following variables: TD $(\mathrm{m} / \mathrm{min}), \operatorname{LSD}(\mathrm{m} / \mathrm{min})$ $\leq 15 \mathrm{~km} / \mathrm{hr}$, HSD $(\mathrm{m} / \mathrm{min})>15 \mathrm{~km} / \mathrm{hr}$, session-RPE, TQR, $\operatorname{mood}$ state vigor, and mood state fatigue.

\section{DISCUSSION}

The present study showed that the TD was markedly lower in the 1st half than in the 2nd half of game, and the same significant difference was observed for LSD in low-intensity displacements and for HSD in high-intensity displacements. These differences were certainly influenced by the substitute players, since according to Mohr et al. (2003), high-intensity displacements are 25\% higher during the last $15 \mathrm{~min}$ of matches in substitute players than in players who started playing. Another preliminary study with a model comparing the times, similar to the present study, was conducted with professional French soccer players and observed greater but not significant distances covered in the second half than in the first due to the substitutions carried out (Carling et al., 2010). In the present study, the decrease in the observed displacements from the first to the second half in entire match players may have occurred due to the development of fatigue during the game (Krustrup et al., 2006).

In the match total average, the results of the TD $(110 \pm 11 \mathrm{~m} /$ $\mathrm{min}$ ) were similar to those of elite Brazilian junior players (109 m/ min), but the study did not report if there were substitutions (Pereira Da Silva et al., 2007). In LSD and HSD actions, the distances $(94 \pm 8 \mathrm{~m} / \mathrm{min}$ and $16 \pm 5 \mathrm{~m} / \mathrm{min}$ ) differ from the findings of the study by Aslan et al. (2012) regarding low intensities (88.1 $\mathrm{m} / \mathrm{min}$ ) and high intensities $(21.9 \mathrm{~m} / \mathrm{min}$ ) for Turkish junior players. With respect to the positions in the game, the present study observed TD characteristics similar to those observed in the study by Saward et al. (2016), in which midfielders covered longer distances than strikers; however, the significance level in the present study was low $(P=0.0755)$. On the other hand, in LSD displacements, midfielders covered significantly longer distances $(P=0.0383$, $P=0.0091)$ than defenders and strikers. Buchheit et al. (2010) found similar results with young soccer players, indicating that defensive midfielders covered longer distances $(P \leq 0.05)$ than strikers. Despite the differences in speed range patterns in these studies, there are tendencies for defenders cover shorter TDs and for midfielders to cover longer TDs in higher speed ranges (Aslan et al., 2012; Buchheit et al., 2010; Mendez-Villanueva et al., 2013; Wehbe et al., 2014). Midfielders likely cover longer distances due to their function of linking the team's defense and attack (Di Salvo et al., 2007). Defenders cover shorter distances because their main purpose is to protect the goal; thus, their displacements are restricted to strategic places in the field (Buchheit et al., 2013). With regard to strikers, many studies (Aslan et al., 2012; Buchheit et al., 2010; 
Wehbe et al., 2014) have shown that they cover shorter distances than players in other positions, a fact that was not confirmed in this study.

Analyzing the differences between the entire match, replaced, and substitute players, it was noted that entire match players presented a significantly lower TD $(P=0.0168, P=0.0068)$ than replaced and substitute players. These results are analogous to those of a study by Bradley et al. (2014) involving English professional soccer players, which concluded that the TD covered by the soccer players who played the entire match $(112 \pm 10 \mathrm{~m} / \mathrm{min})$ was significantly shorter $(P<0.0001)$ than that of replaced $(116 \pm 11 \mathrm{~m} / \mathrm{min})$ and substitute players $(120 \pm 15 \mathrm{~m} / \mathrm{min})$; however, unlike the present study, there was a difference $(P<0.0001)$ between replaced and substitute players. Similar to this work, Bradley et al. (2014) verified the displacements in different speed ranges, identifying that during high intensity $(14.4-19.7 \mathrm{~km} / \mathrm{hr})$, the entire match soccer players covered significantly shorter distances $(P<0.0001)$ than the replaced and substitute players, and the replaced players covered shorter distances $(P<0.0001)$ than the substitute players. These results are similar to those of the present study, which observed significantly shorter HSD displacements $(P=0.0011, P=$ $0.0189)$ among entire match players than among substitute and replaced players. In very low-intensity displacements (0.7-7.1 $\mathrm{km} / \mathrm{hr}$ ), Bradley et al. (2014) noticed that substitute soccer players covered shorter distances $(P<0.0001)$ than the replaced and entire match players, while in low intensities $(7.2-14.3 \mathrm{~km} / \mathrm{hr})$ the entire match and replaced players covered shorter distances $(P<0.0001)$ than the substitute players. The present study used a different low-intensity range $(\leq 15 \mathrm{~km} / \mathrm{hr})$ and verified that there was a smaller but not significant difference $(P=0.0804)$ between the entire match and replaced players alone.

The present study verified that the average value of the TQR in soccer players in seven games was $15.3 \pm 1.0$ in psychophysical scales; in other words, according to the scale used, the athletes reported "good recovery" (Kenttä and Hassmén, 1998). In relation to session-RPE, the values obtained were $569 \pm 289$ AUs, which are inferior values compared with those in the study by Wrigley et al. (2012), in which players presented $759 \pm 51$ AUs in a competitive match with elite junior soccer players. This difference is likely due to average values that were influenced by the substitutions in the matches. In soccer positions, recovery of soccer players does not depend on "good" or "very good" positioning performed on the field according to the scale used (Kenttä and Hassmén, 1998). With respect to the session-RPE, although it did not present significant differences, there was great variation in the values, which ranged between $443 \pm 295$ and $819 \pm 107$; this finding may be related to the positions in which there was a higher number of replaced and substitute players. These data corroborate those of the study by Gomez-Piriz et al. (2011) that involved professional soccer players $(\mathrm{n}=22)$ and evaluated the session-RPE in 13 training sessions and organized the results according to the game positions, with no reports of significant differences among them. Another aspect of the TQR observed in the present study was that the recovery of the entire match players was lower than the recovery of the substitute players $(P=0.0016)$ and the replaced players $(P=0.0384)$. The same happened with the mood state, represented by fatigue, in which the entire match players presented a higher value $(P=0.0460)$ than the substitute players. These altered perceptions and sensations of TQR and fatigue are likely related to the level of anxiety before the matches because the entire match players were already aware of the condition of starters in advance of the line-up. In relation to the session-RPE, the entire match and replaced players reported higher perceived exertion $(P<0.0001)$ than the substitute players, confirming the tendency that the higher the participation time is, the higher the exertion perception (Foster et al., 2001).

Regarding mood states, this study verified that in the average of games the results were similar for vigor (11.8 \pm 3.1$)$ and fatigue $(0.9 \pm 1.2)$. These results show mood states compatible with what was expected in a high-performance competitive sports environment, with a positive mood due to elevated vigor associated with a low level of fatigue (Bertollo et al., 2009). In different positions of the game, no significant differences were observed in mood states for vigor and fatigue among soccer players.

Another important issue regarding substitutions is that all of them took place in the 2nd half, and from 19 substitutions carried out during seven games, only one involved defender; in other words, 18 occurred with midfielders and strikers, showing a tendency to look for performance improvement in the attack line. The same tendency was highlighted by Bradley et al. (2014), who verified that most of the substitutions $(P \leq 0.05)$ took place during the second stage of the match, and the attack lines (midfielders and strikers) were prioritized $(P<0.0001)$. The preference for substitutions of midfielder and striker players possibly occurs for tactical reasons including increasing the creation of opportunities for goal (Bradley et al., 2014). In a study of professional French soccer players ( $\mathrm{n}=18$ ), Carling et al. (2010) noticed that during the first ten minutes as substitute players, midfielders cover distances with high intensity in a similar way to the first ten minutes of the games. In other words, while the team partners and opponents present 
decreased physical performance, the substitute players show great conditions. The coaches must unfailingly carry out substitutions of soccer players to keep and increase the team game intensity, but contextual factors (injuries and cramps), the game model, the team tactical philosophy, and technical, positional and situational factors (Bradley et al., 2014; Hills et al., 2019) as well as the psychophysical and psychological conditions of athletes during the competition are essential in these decisions.

In summary, the present study was able to characterize the demands of displacements of elite junior soccer players throughout one of the biggest national competitions of the category. As a differential, it presented the behavior of psychological states through mood states and psychophysical demands using the TQR and RPE scales. The data reported in this study show important details that regardless of the context, extrapolation of data can be useful for the team competitive strategies. Substitutions are decisive for maintenance and for increasing the total displacement average and players' high intensity, balancing a tendency toward decreased motor performance in the second half of games played by junior soccer players. New studies could analyze the impact of the substitutions of young soccer players.

\section{CONFLICT OF INTEREST}

No potential conflict of interest relevant to this article was reported.

\section{REFERENCES}

Aslan A, Acikada C, Güvenç A, Gören H, Hazir T, Ozkara A. Metabolic demands of match performance in young soccer players. J Sports Sci Med 2012;11:170-179.

Bertollo M, Saltarelli B, Robazza C. Mental preparation strategies of elite modern pentathletes. Psyc Sports Exerc 2009;10:244-254.

Borg GA. Psychophysical bases of perceived exertion. Med Sci Sports Exerc 1982;14:377-381.

Bradley PS, Lago-Peñas C, Rey E. Evaluation of the match performances of substitution players in elite soccer. Int J Sports Physiol Perform 2014; 9:415-424.

Brandt R, Herrero D, Massetti T, Crocetta TB, Guarnieri R, Monteiro CBM, Viana MS, Bevilacqua GG, Abreu LC, Andrade A. The Brunel Mood Scale rating in mental health for physically active and apparently healthy populations. Health 2016;8:125-132.

Buchheit M, Mendez-Villanueva A, Simpson BM, Bourdon PC. Match running performance and fitness in youth soccer. Int J Sports Med

\section{0;31:818-825}

Buchheit M, Simpson BM, Mendez-Villanueva A. Repeated high-speed activities during youth soccer games in relation to changes in maximal sprinting and aerobic speeds. Int J Sports Med 2013;34:40-48.

Carling C, Espié V, Le Gall F, Bloomfield J, Jullien H. Work-rate of substitutes in elite soccer: a preliminary study. J Sci Med Sport 2010;13:253255.

Cummins C, Orr R, O'Connor H, West C. Global positioning systems (GPS) and microtechnology sensors in team sports: a systematic review. Sports Med 2013;43:1025-1042.

Di Salvo V, Baron R, Tschan H, Calderon Montero FJ, Bachl N, Pigozzi F. Performance characteristics according to playing position in elite soccer. Int J Sports Med 2007;28:222-227.

Fanchini M, Ghielmetti R, Coutts AJ, Schena F, Impellizzeri FM. Effect of training-session intensity distribution on session rating of perceived exertion in soccer players. Int J Sports Physiol Perform 2015;10:426430.

Foster C, Florhaug JA, Franklin J, Gottschall L, Hrovatin LA, Parker S, Doleshal $\mathrm{P}$, Dodge $\mathrm{C}$. A new approach to monitoring exercise training. J Strength Cond Res 2001;15:109-115.

Gomez-Piriz PT, Jiménez-Reyes P, Ruiz-Ruiz C. Relation between total body load and session rating of perceived exertion in professional soccer players. J Strength Cond Res 2011;25:2100-2103.

Hills SP, Barrett S, Feltbower RG, Barwood MJ, Radcliffe JN, Cooke CB, Kilduff LP, Cook CJ, Russell M. A match-day analysis of the movement profiles of substitutes from a professional soccer club before and after pitch-entry. PLoS One 2019;14:e0211563.

Hills SP, Barwood MJ, Radcliffe JN, Cooke CB, Kilduff LP, Cook CJ, Russell M. Profiling the responses of soccer substitutes: a review of current literature. Sports Med 2018;48:2255-2269.

Kenttä G, Hassmén P. Overtraining and recovery. A conceptual model. Sports Med 1998;26:1-16.

Krustrup P, Mohr M, Steensberg A, Bencke J, Kjaer M, Bangsbo J. Muscle and blood metabolites during a soccer game: implications for sprint performance. Med Sci Sports Exerc 2006;38:1165-1174.

Mendez-Villanueva A, Buchheit M, Simpson B, Bourdon PC. Match play intensity distribution in youth soccer. Int J Sports Med 2013;34:101110.

Mohr M, Krustrup P, Bangsbo J. Match performance of high-standard soccer players with special reference to development of fatigue. J Sports Sci 2003;21:519-528.

Pereira Da Silva N, Kirkendall DT, Leite De Barros Neto T. Movement patterns in elite Brazilian youth soccer. J Sports Med Phys Fitness 2007; 47:270-275.

Saward C, Morris JG, Nevill ME, Nevill AM, Sunderland C. Longitudinal 
development of match-running performance in elite male youth soccer players. Scand J Med Sci Sports 2016;26:933-942.

Slimani M, Bragazzi NL. Data concerning the impact of mental fatigue on vigor as measured with the Brunel Mood Scale (BRUMS) in both physically active and trained subjects: a mini meta-analysis. Data Brief 2017;13:655-660.

Turner AN, Stewart PF. Strength and conditioning for soccer players.
Strength Cond J 2014;36:1-13.

Wehbe GM, Hartwig TB, Duncan CS. Movement analysis of Australian national league soccer players using global positioning system technology. J Strength Cond Res 2014;28:834-842.

Wrigley R, Drust B, Stratton G, Scott M, Gregson W. Quantification of the typical weekly in-season training load in elite junior soccer players. J Sports Sci 2012;30:1573-1580. 